\title{
A Two-component Transport Model for Solar Wind Fluctuations: Waves plus Quasi-2D Turbulence
}

\author{
Sean Oughton*, William H. Matthaeus ${ }^{\dagger}$, Charles W. Smith** and Ben Breech ${ }^{\ddagger}$ \\ * Department of Mathematics, University of Waikato, Hamilton 3240, New Zealand \\ ${ }^{\dagger}$ Bartol Research Institute and Department of Physics \& Astronomy, University of Delaware, Newark DE 19716 \\ ${ }^{* *}$ Institute for Earth, Oceans and Space, University of New Hampshire, Durham NH 03824 \\ ${ }^{\ddagger}$ NASA Goddard Space Flight Center, Greenbelt MD 20771
}

\begin{abstract}
.
We present a model for the transport of solar wind fluctuations, based on the assumption that they can be well-represented using two distinct components: a quasi- $2 D$ turbulence piece and a wave-like piece. For each component, coupled transport equations for its energy, cross helicity, and characteristic lengthscale(s) are derived, along with an equation for the proton temperature. This energy-containing "two-component" model includes the effects of solar wind expansion and advection, driving by stream shear and pickup ions, and nonlinear cascades. Nonlinear effects are modeled using a recently developed one-point phenomenology for such a two-component model of homogeneous MHD turbulence [1]. Heating due to these nonlinear effects is included in the temperature equation. Numerical solutions are discussed and compared with observations.
\end{abstract}

\section{BACKGROUND}

Spatial transport of MHD-scale fluctuations in the solar wind has been investigated for over 40 years. It is, however, yet to be completely understood. Even in the simplest case of radial transport there are numerous effects that may need to be represented to obtain accord with observations. These include wave and turbulence activity, forcing processes (e.g., stream shear, pickup ion driving), and the spatial and spectral anisotropy of the fluctuations.

Studies which take into account some or all of these processes have appeared in the literature [e.g., 2-13], with useful reviews available [13-15]. Many of these are concerned with the transport of quantities based on energy-containing scales, e.g., rms values of the fluctuation fields and their characteristic lengthscales. Moreover, they often approximate the fluctuations as being of a single kind, e.g., only Alfvén waves or only turbulence.

However, there are multiple lines of support for the presence — and relevance — of at least two distinct types of fluctuations. For example, from early solar wind observations it was inferred that both turbulence features [16] and wave features [17] were present. Subsequent support has come from numerous observational studies [18-27], theory [28-32], and simulation studies [e.g., 33-36]. Furthermore, the physics of how the fluctuations are driven is of at least two types: (stream) shear drives low-frequency fluctuations, while pickup ions inject energy at much higher frequencies and shorter lengthscales. It is clearly problematic to address both types of driving consistently with only a single component. Thus, it would seem appropriate, and advantageous, to develop an energy-containing style model for the transport of solar wind fluctuations which allows for distinct types of fluctuations. We present such a "two-component" model below. A major advantage of this approach is that it yields much improved radial profiles for the correlation lengths, as compared with observations. (Note that models for the spatial transport of spectra rather than energycontaining range quantities, typically do include two (or more) components $[14,37-41]$.)

\section{DEVELOPMENT OF THE MODEL}

\subsection{Two-component turbulence}

A key ingredient for a two-component transport theory is a suitable model for the nonlinear interactions of the two components. A recently developed energycontaining phenomenology for homogeneous MHD turbulence with a mean magnetic field [1] provides this element. It models the fluctuations as two interacting components, conveniently (if not quite accurately) referred to as quasi-2D and wave-like. Alternative designations are the low-frequency and high-frequency pieces. Each component is represented by its energy, cross helicity, and characteristic lengthscale (perpendicular to the mean magnetic field). Evolving the wave-like component also requires knowledge of its characteristic parallel scale, $\lambda_{\|}$. We recall the form of this phenomenology before proceeding to the full transport model.

Denote the quasi-2D energy as $Z^{2} / 2$, the wave-like energy as $W^{2} / 2$, and their respective perpendicular length-

CP1216, Twelfth International Solar Wind Conference,

edited by M. Maksimovic, K. Issautier, N. Meyer-Vernet, M. Moncuquet, and F. Pantellini

(C) 2010 American Institute of Physics 978-0-7354-0759-6/10/\$30.00 
scales as $\ell$ and $\lambda$. The simplest version of the phenomenology is the zero cross helicity case [1]:

$$
\begin{aligned}
\frac{\mathrm{d} Z^{2}}{\mathrm{~d} t} & =-\frac{Z^{3}}{\ell}-\frac{W Z^{2}}{\ell} \frac{2}{1+Z / W}+X, \\
\frac{\mathrm{d} W^{2}}{\mathrm{~d} t} & =-\frac{Z W^{2}}{\lambda} \frac{2}{1+\lambda / \ell}-\frac{2 W^{4}}{\lambda^{2}} \frac{\lambda_{\|}}{V_{A}}-X,
\end{aligned}
$$

Here, $V_{A}$ is the Alfvén speed associated with the uniform magnetic field, and $X=2 W Z\left(W^{2}+Z^{2}\right)\left(\frac{1}{\lambda^{2}}-\frac{1}{\ell^{2}}\right) \frac{\lambda_{\|}}{V_{A}}$ is the "exchange" term between the two components, which can be positive or negative. The other RHS terms are also readily identified. In (1), the first term is a Kármán-Taylor model [42] for the self-decay of the $Z$ component (the energy-containing partner to the Kolmogorov cascade), while the second term represents decay of $Z$ due to $Z-W$ interactions. Similarly, in (2) the first term models the resonant perpendicular cascade $[43,44]$, sometimes referred to as weak turbulence $[45,46]$. Note that it requires $Z \neq 0$. The second represents a Kraichnan-like cascade [47, 48]. Except for the latter, these correspond to (approximately) perpendicular cascades. Typically, the leading-order term in each equation is the first one on the RHS [1].

For the more general situation of non-zero cross helicities, as needed for the solar wind, the equations are similar with each term being multiplied by cross helicitydependent attenuation factors, denoted generically as $f$ [11]. The essential points are that the structure is unchanged but the nonlinearities are weakened.

\subsection{Transport Model}

Solar wind fluctuations evolve due to the effects of wind expansion and advection, stream shear, nonlinear interactions, and, in the outer heliosphere, pickup ion driving. (See [13] for an up-to-date summary and details of the approach employed below.) The linear portions of the transport equations for general fluctuations are readily derived $[13,37,38]$. The starting point is the MHD equations subject to an assumption of scale-separation between the fluctuations and the large-scale fields. The Elsässer fluctuations are then decomposed into quasi2D and wave-like pieces. The steps and approximations made thereafter are as documented in earlier works [e.g., $11,13]$, along with use of orthogonality properties of products of the two components (when averaged over the small scales) [1]. Replacing the nonlinear terms with the generalization of (1)-(2), yields steady-state equations for the energies ( $T$ is plasma temperature, and $m_{p}$ proton mass):

$$
\frac{\mathrm{d} Z^{2}}{\mathrm{~d} r}=-\left[1+M \sigma_{D}-C_{\mathrm{sh}}^{Z}\right] \frac{Z^{2}}{r}-\frac{\alpha f}{U} \frac{Z^{3}}{\ell}+\frac{\alpha f_{X}}{U} X,
$$

$$
\begin{aligned}
\frac{\mathrm{d} W^{2}}{\mathrm{~d} r}= & -\left[1+M \tilde{\sigma}_{D}-C_{\mathrm{sh}}^{W}\right] \frac{W^{2}}{r}-\frac{\tilde{\alpha} \tilde{f}}{U} \frac{Z W^{2}}{\lambda} \frac{2}{1+\lambda / \ell} \\
& -\frac{\alpha f_{X}}{U} X+\frac{\dot{E}_{P I}}{U}, \\
\frac{\mathrm{d} T}{\mathrm{~d} r}= & -\frac{4 T}{3 r}+\frac{m_{p}}{3 U k_{B}}\left[\alpha f \frac{Z^{3}}{\ell}+\frac{Z W^{2}}{\lambda} \frac{2 \tilde{\alpha} \tilde{f}}{1+\lambda / \ell}\right] .(5)
\end{aligned}
$$

Here $r$ is heliocentric distance and $U$ the uniform solar wind speed. (See [7, 9] for considerations regarding variable solar wind speed.) For clarity, only the leading-order and exchange terms from the non-zero cross helicity version of (1)-(2) have been written here. (When computing the numerical solutions, all terms are included.)

The notation is based on that used in earlier, singlecomponent, transport models [12, 13]. For the quasi$2 \mathrm{D}$ piece, $\sigma_{c}$ is the normalized cross helicity, $\sigma_{D}$ the normalized energy difference, and $\alpha, \beta$ are $O(1)$ fitting constants. Tildes indicate the analogous quantity for the wave-like component. The strength of the large-scale shear driving is controlled with the constants $C_{\mathrm{sh}}, \hat{C}_{\mathrm{sh}}$, the mixing tensor $M$ represents large-scale gradients of the large-scale fields, and $\dot{E}_{P I}$ is the pickup ion driving, approximated as in earlier works [e.g., 5, 6, 11, 12]. Only a small fraction of the 'available' pickup ion energy ends up being transferred to the wave-like component [10]. The attenuation factors, $f, f_{X}, f^{\prime}$, etc., depend upon one or both of $\sigma_{c}$ and $\tilde{\sigma}_{c}$; all are bounded by \pm 1 [11].

The approach also yields lengthscale equations,

$$
\begin{gathered}
\frac{\mathrm{d} \ell}{\mathrm{d} r}=\left[M \sigma_{D}-\hat{C}_{\mathrm{sh}}^{Z}\right] \frac{\ell}{r}+\frac{\beta}{U}\left[f Z-f_{X} X \frac{\ell}{Z^{2}}\right] \\
\frac{\mathrm{d} \lambda}{\mathrm{d} r}=\left[M \tilde{\sigma}_{D}-\hat{C}_{\mathrm{sh}}^{W}\right] \frac{\lambda}{r}+\frac{\tilde{\beta}}{U}\left[\tilde{f} Z+f_{X} X \frac{\alpha \lambda}{\tilde{\alpha} W^{2}}\right] \\
\frac{\mathrm{d} \lambda_{\|}}{\mathrm{d} r}=\left[M \tilde{\sigma}_{D}-\hat{C}_{\mathrm{sh}}^{W}\right] \frac{\lambda_{\|}}{r}-\left(\lambda_{\|}-\lambda_{\mathrm{res}}\right) \frac{\dot{E}_{P I}}{U W^{2}}+\frac{2 f_{\mathrm{Kr}} W^{2} \lambda_{\|}^{2}}{U V_{A} \lambda^{2}}
\end{gathered}
$$

and equations for the normalized cross helicities,

$$
\begin{aligned}
\frac{\mathrm{d} \sigma_{c}}{\mathrm{~d} r} & =\alpha f^{\prime} \frac{Z}{\ell U}-\left[\frac{C_{\mathrm{sh}}^{Z}-M \sigma_{D}}{r}\right] \sigma_{c} \\
\frac{\mathrm{d} \tilde{\sigma}_{c}}{\mathrm{~d} r} & =\tilde{\alpha} \tilde{f}^{\prime} \frac{Z}{\lambda U} \frac{2}{1+\lambda / \ell}-\left[\frac{C_{\mathrm{sh}}^{W}-M \tilde{\sigma}_{D}}{r}+\frac{\dot{E}_{P I}}{U W^{2}}\right] \tilde{\sigma}_{c}
\end{aligned}
$$

where again for clarity some higher-order terms are not written. The additional quantity $\lambda_{\text {res }}$ is the (parallel) wavenumber of the waves resonant with the pickup ions. We employ the functional form $\lambda_{\text {res }} \sim r$, as developed in [10]. In the lengthscale equations, the forms of the nonlinear terms (the $\beta, \tilde{\beta}$ ones) arise from imposition of conservation laws [see 1, 13, 49].

Several further features of the two-component model warrant discussion. As noted above, pickup ion driving occurs at high frequencies $[8,10]$, and hence should appear in the $W^{2}$ equation (and not in the $Z^{2}$ one), as is the 

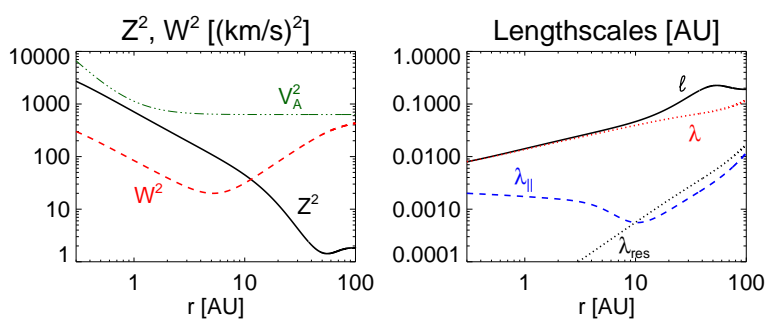

FIGURE 1. (color online) Sample solutions with $U=$ $380 \mathrm{~km} / \mathrm{s}, M \sigma_{D}=M \tilde{\sigma}_{D}=-\frac{1}{6}, C_{\mathrm{sh}}^{Z, W}=1, \hat{C}_{\mathrm{sh}}^{Z, W}=0, \alpha=2 \beta=$ $\frac{1}{4}$. Boundary conditions may be read off the left-hand scales.

case herein. In single-component models, this physical distinction is difficult to make. In fact, it is even more important in connection with the lengthscales: Pickup ions are thought to interact with the parallel wavenumber of the fluctuations, injecting energy at that scale [10], perhaps subject to a conservation law connecting $W^{2}$ and $\lambda_{\|}$. In transport models without a $\lambda_{\|}$, the physics is incorporated incorrectly since the driving occurs instead at the correlation scale, which has lead to difficulties in matching observations $[5,6,8]$. Numerical solutions of the present model produce correlation lengths which are in better accord with observations (see below).

As far as heating of the fluctuations is concerned, the various turbulent cascades discussed after (2) all contribute, as seen in the square-bracketed term in (5).

Ostensibly, moving from a one- to a two-component model for solar wind fluctuations has introduced considerably more complexity. There are now eight equations to solve instead of four, and the number of nonlinear terms is also much larger. From a numerical perspective, however, this is still a small system of well-behaved ordinary differential equations and thus is no harder to solve than the single-component models used previously.

\subsection{Numerical Solutions}

We now discuss a representative solution to the twocomponent model, using parameters appropriate for the ecliptic, and compare it with observational data. Note that this solution is not an optimized fit to the data. Thus, we anticipate that the already encouraging agreement can be significantly improved. Solutions were obtained using a standard fourth-order Runge-Kutta package.

Figure 1 displays the energies and lengthscales of the components. Injection of pickup ion energy into the wave-like component is evident. The correlation lengths $\ell$ and $\lambda$ are not greatly affected by the pickup ions, unlike the parallel scale $\lambda_{\|}$, which tracks just under $\lambda_{\text {res }}$ for $r \gtrsim 10 \mathrm{AU}$. The bump in $\ell$ and flattening in $Z^{2}$ near $50 \mathrm{AU}$ are due to the exchange term $X$ becoming significant.
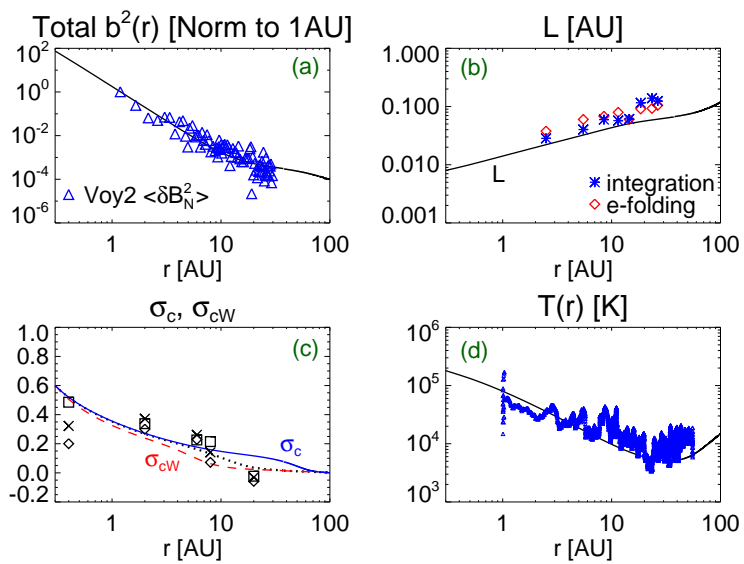

FIGURE 2. (color online) Model solutions (curves) and Voyager observational data (symbols). See [6] for details on the observational data. In (c), the dotted curve is the composite $\sigma_{c}$ and observational values are from Helios and Voyager [50].

Figure 2 shows results from the two-component model in forms suitable for comparison with observations. Panel (a) plots the total magnetic energy in the two components normalized to its value at $1 \mathrm{AU}$. The agreement with the ' $\mathrm{N}$ ' component of Voyager magnetic field RTN data is of a similar quality to single-component models [e.g., 5, 6]. Similar comments hold for the temperature [panel (d)]. For the correlation length, we plot the composite "centre of mass"-style quantity $L=\left(Z^{2} \ell+\right.$ $\left.W^{2} \lambda\right) /\left(Z^{2}+W^{2}\right)$, as $\ell$ and $\lambda$ are difficult to obtain observationally. The model solution for $L$ is in good agreement with the data, and is a significant improvement to the behavior seen in single-component models $[5,6,8]$.

Figure 2(c) displays $\sigma_{c}$ and $\tilde{\sigma}_{c}$ and their composite form, defined analogously to $L$. The latter is a reasonable fit to the data points. We recall that $1 \mathrm{AU}$ results indicate the normalized cross helicity is approximately isotropic with respect to the mean magnetic field direction [21], implying that a two-component model should have $\sigma_{c} \approx$ $\tilde{\sigma}_{c}$. For the solution shown, this is true near $1 \mathrm{AU}$, but not for 5-40 AU. Clearly, further study is warranted from both the modeling and observational sides.

\section{CONCLUSIONS}

We have presented an (energy-containing) twocomponent transport model for solar wind fluctuations, with the components corresponding to (a) quasi-2D turbulence, and (b) wave-like fluctuations. Allowing for separate energies and lengthscales for these components enables the physics of shear driving and pickup ion driving to enter more realistically than in single-component versions. Partitioning of the turbulent energy into two 
components leads to several distinct energy cascades. However, as the total energy cascaded is approximately the same as in single-component models, the heating is also largely unchanged and remains consistent with observations.

Numerical solutions afford good agreement with observations of proton temperature, fluctuation energy, cross helicity, and correlation length. The numerical solutions have yet to be optimized. We anticipate that the model will be of use in various space physics applications, including scattering of energetic particles.

Insightful conversations with P. Isenberg are gratefully acknowledged. Research supported by NSF SHINE Program ATM0752135, NASA Heliophysics Theory Program NNX08AI47G (WHM), NASA Sun-Earth Connection Guest Investigator Grant NNX08AJ19G (CWS), and an appointment to the NASA Postdoctoral Program (BAB).

\section{REFERENCES}

1. S. Oughton, W. H. Matthaeus, and P. Dmitruk, Phys. Plasmas 13, 042306 (2006).

2. W. H. Matthaeus, S. Oughton, D. Pontius, and Y. Zhou, J. Geophys. Res. 99, 19267-19287 (1994).

3. G. P. Zank, W. H. Matthaeus, and C. W. Smith, J. Geophys. Res. 101, 17 093-17 107 (1996).

4. W. H. Matthaeus, G. P. Zank, and S. Oughton, J. Plasma Phys. 56, 659-675 (1996).

5. W. H. Matthaeus, G. P. Zank, C. W. Smith, and S. Oughton, Phys. Rev. Lett. 82, 3444-3447 (1999).

6. C. W. Smith, W. H. Matthaeus, G. P. Zank, N. F. Ness, S. Oughton, and J. D. Richardson, J. Geophys. Res. 106, 8253-8272 (2001).

7. C. W. Smith, P. A. Isenberg, W. H. Matthaeus, and J. D. Richardson, Astrophys. J. 638, 508-517 (2006).

8. P. A. Isenberg, C. W. Smith, and W. H. Matthaeus, Astrophys. J. 592, 564-573 (2003).

9. P. A. Isenberg, C. W. Smith, W. H. Matthaeus, and J. D. Richardson, "Turbulent Heating of the Distant Solar Wind by Interstellar Pickup Protons with a variable solar wind speed," in Proc. Solar Wind 11 - Soho 16, ed. B. Fleck, T. Zurbuchen, and H. Lacoste, ESA, Noordwijk, The Netherlands, 2005, vol. SP-592, pp. 346-350.

10. P. A. Isenberg, Astrophys. J. 623, 502-510 (2005).

11. W. H. Matthaeus, J. Minnie, B. Breech, S. Parhi, J. W. Bieber, and S. Oughton, Geophys. Res. Lett. 31, L12803, doi:10.1029/2004GL019645 (2004).

12. B. Breech, W. H. Matthaeus, J. Minnie, S. Oughton, S. Parhi, J. W. Bieber, and B. Bavassano, Geophys. Res. Lett. 32, L06103, doi:10.1029/2004GL022321 (2005).

13. B. Breech, W. H. Matthaeus, J. Minnie, J. W. Bieber, S. Oughton, C. W. Smith, and P. A. Isenberg, J. Geophys. Res. 113, A08105, doi:10.1029/2007JA012711 (2008).

14. C.-Y. Tu and E. Marsch, Space Sci. Rev. 73, 1 (1995).

15. R. Bruno, B. Bavassano, R. D’Amicis, V. Carbone, L. Sorriso-Valvo, and E. Pietropaolo, Space Sci. Rev. 122, 321 (2006).

16. P. J. Coleman, Astrophys. J. 153, 371-388 (1968).
17. J. W. Belcher, and L. Davis Jr., J. Geophys. Res. 76, 3534-3563 (1971).

18. W. H. Matthaeus, M. L. Goldstein, and D. A. Roberts, $J$. Geophys. Res. 95, 20 673-20 683 (1990).

19. J. W. Bieber, W. Wanner, and W. H. Matthaeus, J. Geophys. Res. 101, 2511-2522 (1996).

20. C. W. Smith, "The Geometry of Turbulent Magnetic Fluctuations at High Heliographic Latitude," in Solar Wind Ten, ed. M. Velli, R. Bruno, and F. Malara, AIP, Melville, NY, 2003, vol. 679, pp. 413-416.

21. L. J. Milano, S. Dasso, W. H. Matthaeus, and C. W. Smith, Phys. Rev. Lett. 93, 155005 (2004).

22. S. Dasso, L. J. Milano, W. H. Matthaeus, and C. W. Smith, Astrophys. J. 635, L181-L184 (2005).

23. K. Hamilton, C. W. Smith, B. J. Vasquez, and R. J. Leamon, J. Geophys. Res. 113, A01106, doi:10.1029/2007JA012559 (2008).

24. J. A. Tessein, C. W. Smith, B. T. MacBride, W. H. Matthaeus, M. A. Forman, and J. E. Borovsky, Astrophys. J. 692, 684-693 (2009).

25. T. Horbury, M. A. Forman, and S. Oughton, Plasma Phys. Controlled Fusion 47, B703-B717 (2005).

26. T. S. Horbury, M. Forman, and S. Oughton, Phys. Rev. Lett. 101, 175005 (2008).

27. J. J. Podesta, Astrophys. J. 698, 986-999 (2009).

28. H. R. Strauss, Phys. Fluids 19, 134-140 (1976).

29. D. C. Montgomery, Phys. Scr. T2/1, 83-88 (1982).

30. G. P. Zank, and W. H. Matthaeus, J. Plasma Phys. 48, 85-100 (1992).

31. J. C. Higdon, Astrophys. J. 285, 109-123 (1984).

32. P. Goldreich, and S. Sridhar, Astrophys. J. 438, 763 (1995)

33. S. Ghosh, W. H. Matthaeus, D. A. Roberts, and M. L. Goldstein, J. Geophys. Res. 103, 23 691-23 704 (1998).

34. S. Ghosh, W. H. Matthaeus, D. A. Roberts, and M. L. Goldstein, J. Geophys. Res. 103, 23 705-23 715 (1998).

35. J. Maron and P. Goldreich, Astrophys. J. 554, 1175 (2001).

36. P. Dmitruk, W. H. Matthaeus, and S. Oughton, Phys. Plasmas 12, 112304 (2005).

37. Y. Zhou, and W. H. Matthaeus, J. Geophys. Res. 95, 10291 (1990).

38. C.-Y. Tu and E. Marsch, J. Geophys. Res. 98, 1257 (1993).

39. E. Marsch and C.-Y. Tu, J. Geophys. Res. 95, 8211 (1990).

40. E. Marsch and C.-Y. Tu, J. Geophys. Res. 98,21045(1993)

41. S. Oughton, and W. H. Matthaeus, J. Geophys. Res. 100, 14 783-14 799 (1995).

42. T. de Kármán, and L. Howarth, Proc. Roy. Soc. London Ser. A 164, 192-215 (1938).

43. J. V. Shebalin, W. H. Matthaeus, and D. Montgomery, J. Plasma Phys. 29, 525-547 (1983).

44. A. Bondeson, Phys. Fluids 28, 2406-2411 (1985).

45. S. Galtier, S. V. Nazarenko, A. C. Newell, and A. Pouquet, J. Plasma Phys. 63, 447-488 (2000).

46. S. Galtier, S. V. Nazarenko, A. C. Newell, and A. Pouquet, Astrophys. J. 564, L49-L52 (2002).

47. R. S. Iroshnikov, Astron. Zh. 40, 742 (1963) [Sov. Astron. 7, 566-571 (1964)].

48. R. H. Kraichnan, Phys. Fluids 8, 1385-1387 (1965).

49. M. Hossain, P. C. Gray, D. H. Pontius Jr., W. H. Matthaeus, and S. Oughton, Phys. Fluids 7, 2886 (1995).

50. D. A. Roberts, M. L. Goldstein, L. W. Klein, and W. H. Matthaeus, J. Geophys. Res. 92, 12 023-12 035 (1987). 